\title{
High Speed SPM for Novel Property Mapping of Functional Ceramics
}

\author{
V. Vyas ${ }^{1}$, V. Palumbo ${ }^{1}$, J. Bosse $^{1}$, Y. Kutes ${ }^{1}$, L. Ye $^{1}$, and B. D. Huey ${ }^{1}$ \\ ${ }^{1}$ University of Connecticut, Materials Science and Engineering Department, Storrs, CT, USA
}

Functional ceramics are crucial to a wide range of advanced technologies, including sensors and actuators, power generation and storage, computing and data storage, etc. The incessant demand for enhanced performance in smaller and lower-power packages necessitates a thorough understanding of these systems, typically at the micro- to nano-scale. In ceramics, this is often especially true at interfaces, which can dominate device behavior and hence provide opportunities for engineering improved or new functionalities. Corresponding measurements of materials properties with the requisite spatial and temporal resolution are challenging, however, particularly during in situ device operation. Accordingly, a high speed variation of Atomic Force Microscopy (AFM) known as 'HSSPM' has been developed to investigate functional materials properties.

HSSPM operates similar to standard AFM, detecting interactions between a scanned surface and a sharp probe with an integrated cantilever. Scan speeds as fast as $2 \mathrm{~cm} / \mathrm{s}$ have been achieved, implementing line rates up to $2 \mathrm{kHz}$, allowing standard $256 \times 256$ pixel im ages to be acquired at up to 8 frames/s. This is based on commercial AFM hardware, but requires high speed detectors and data acquisition. Furthermore, it necessitates automated system control to achieve the high acquisition rates, sequentially adjust experimental parameters from one im age to the next, and manage the substantially greater saved data than conventional AFM. Automated image analysis is also crucial for handling the volume of results. For imaging modes benefitting from AC oscillations, contact resonances are also employed.

In this manner, of course high throughput imaging is feasible. The primary application, though, has been to monitor dynamic processes by im aging as a function of time, often during in situ excitation with additional stimuli. Other approaches leverage the ability to perform rapid measurements in a precise and hence with minimal thermal drift. Repeating this process with simple feature tracking from one frame to another, a material's local response to a wide range of distinct stim uli (temperature, voltage, magnetic field, time, etc.) has been mapped. Im aging modes are demonstrated including topography, friction, piezoresponse, contact resonance, current, electric fields, and magnetic fields.

As an example, Fig. 1 displays high speed Piezo-Force-Microscopy images (hs-pfm ) for an epitaxial PZT thin film, revealing dom ain walls (top) between domains oppositely oriented either into or out of the plane of the film (base). A sequence of 256 such images, each acquired with a sequentially $\mathrm{m}$ ore positive (or negative) simultaneously app lied DC bias $\left(\mathrm{V}_{\mathrm{o}}\right)$, reveals the dynam ics of the switching process from outward to inwardly poled domains (Fig. 2). The average contras $t$ for each such im age (8 are shown) provides a macroscopic measure of the mean domain state for the $2 \times 2 \mu \mathrm{m}^{2}$ area measured, which when plotted against the applied bias provides a classic ferroelectric hysteresis loop. Crucially, however, this loop is resolved at the nanoscale, allowing mapping not just of the overall response for a device of such dimensions, but also the local nucleation times, nucleation sequence, domain wall velocities, domain wall directionality, etc. This response is absolutely repeatable, and hence is not stochastic but rather results from a distribution of defects within the specimen. The method therefore enables mapping the defect-defined energy landscape for switching. Similar results have been achieved in response to voltage pulses with durations down to $10 \mathrm{nsec}$. These experiments resolved enhancements 
in domain growth rates of more than $10 \times$ until the domain size expands to $\sim 100 \mathrm{~nm}$, far enough from the defect site that the expanding domain wall has climbed out of the potential well in which it nucleated.

Other examples being presented of nanoscale ceramic functional properties include:

- HSSPM to map photovoltaic performance measures for polycrystalline CdS solar cells and solid state Graetzel style photovoltaics;

- Direct evidence of solid state diffusion of Ag ions from nanoparticles into chalcogenide glass films;

- Voltage and time thresholds for switching in resistive RAM devices;

- Forces and resonant modes of MEMS devices during operation.

References:

[1] The authors acknowledge funding from the Department of Energy, Basic Energy Sciences, Electron and Scanning Probe Microscopies, grant DE-SC-0005037.

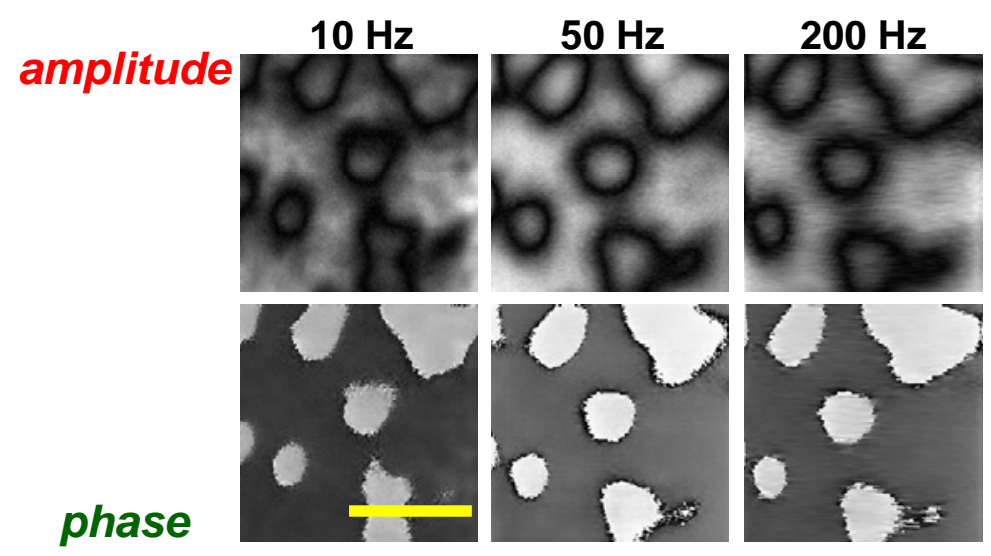

Figure 1. Ferroelectric dom ain walls (top, hs-pfm amplitude) and do main orientations (base, hs-pfm phase) imaged $10 \times, 50 \times$, and $100 \times$ faster than normal. $250 \mathrm{~nm}$ scale bar shown.

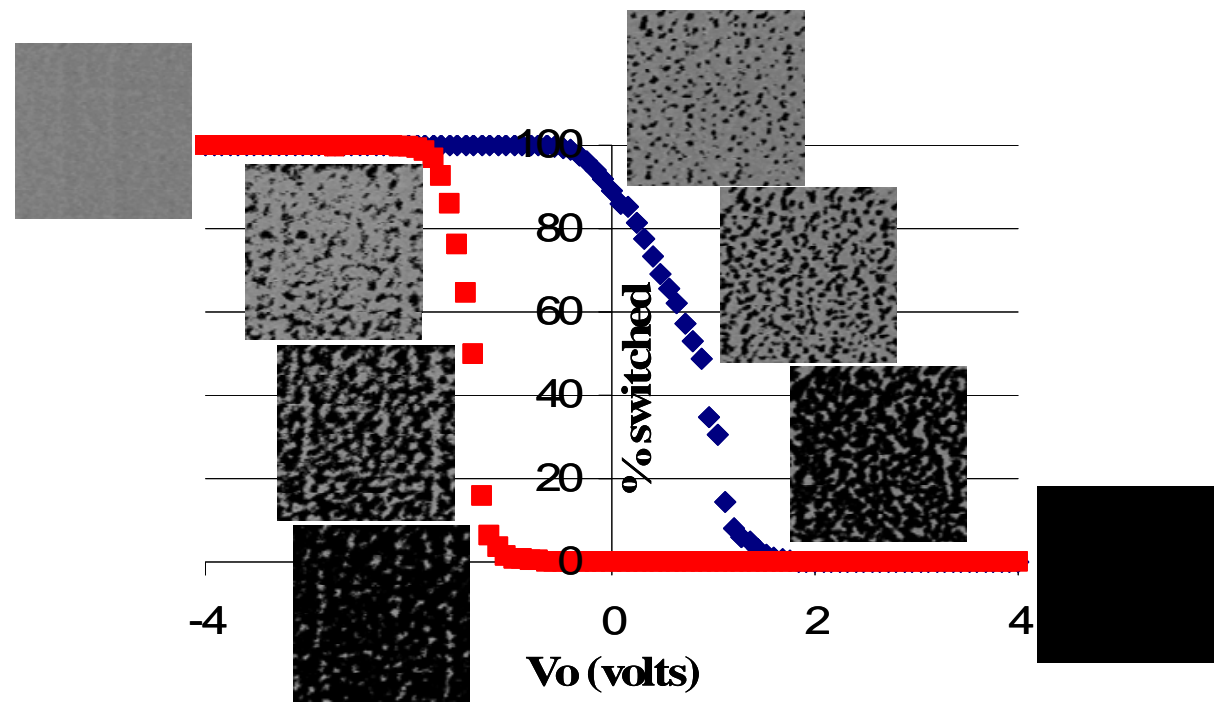

Figure 2. Ferroelectric hysteresis loop resolved by hs-pfm imaging of a $2 \times 2 \mu \mathrm{m}^{2}$ area with simultaneously applied DC offset biasing $\left(\mathrm{V}_{\mathrm{o}}\right)$, resolving the nucleation tim e, energy, order, and pattern which ultimately provides the defect-defined energy landscape for switching. 